\title{
Es confirma la presència de Synchita undata Guérin-Méneville, 1844 (Zopheridae) a la península Ibèrica i es donen noves o interessants citacions de coleòpters per a Catalunya (Coleoptera)
}

\author{
Amador Viñolas \\ Josep Muñoz-Batet \\ Joaquim Soler \\ Consorci del Museu de Ciències Naturals de Barcelona. Laboratori de Natura \\ Col-lecció d'Artròpodes \\ Passeig Picasso, s/n. 08003 Barcelona \\ av.rodama@gmail.com
}

\section{Resum}

Les campanyes realitzades en el Paratge d'Interès Nacional de l'Albera (Girona) des de fa 14 anys i en el de Poblet (Tarragona) durant els anys 2012 i 2013, per a l'estudi de la biodiversitat de coleòpters, han aportat un gran nombre de novetats per a la península Ibèrica i per a Catalunya, moltes d'elles ja comunicades en anteriors treballs.

En la present nota es confirma Synchita undata Guérin-Méneville, 1884 a la península Ibèrica, i se citen 14 espècies noves o amb poques referències per a Catalunya i 19 que no estaven citades de Tarragona.

També es donen dades sobre la biologia dels Dermestidae, Orphilus niger (Rossi, 1790) i O. beali Zhantiev, 2001, Se'n comenta la seva distribució i es representa el seu edeagus, únic element segur per poder diferenciar les dues espècies.

Paraules clau: coleòptera; nous registres; península Ibèrica; Catalunya.

Abstract. The occurrence of Synchita undata Méneville-Guérin, 1844 (Zopheridae) is confirmed in the Iberian Peninsula and new or interesting records for Catalonia beetles (Coleoptera) are given

The campaigns carried out for the study of biodiversity of coleoptera fauna in the Paratge d'Interès Nacional de l'Albera (Girona) over 14 years and in the campaign of Poblet (Tarragona) in 2012 and 2013 have contributed many new records for the Iberian Peninsula and Catalonia, many of which have already been reported in previous works. The present work confirms the occurrence of Synchita undata Guérin-Méneville, 1884, in the Iberian Peninsula, cites 14 new species or with few references for Catalonia, and 19 that were not previously recorded for Tarragona. Data on the biology of the Dermestidae beetles Orphilus niger (Rossi 
1790) and O. beali Zhantiev, 2001, are also given, with comments on their distribution. Finally, edeagus of both species are represented; the only element to distinguish the two species.

Keywords: Coleoptera; new records; Iberian Peninsula and Catalonia.

\section{Introducció}

S'han continuat els estudis sobre la biodiversitat de coleòpters en el Paratge Natural d'Interès Nacional de l'Albera (Girona), que es duen a terme des de l'any 2000 (Viñolas et al., 2012). Així mateix, a l'àrea del Tillar, en el Paratge Natural d'Interès Nacional de Poblet (serra de Prades, Tarragona), durant l'any 2012 es va realitzar un estudi per conèixer la relació dels coleòpters amb la mortalitat del pi roig (Pinus sylvestris L.) a les muntanyes de Prades, amb la instal-lació de paranys d'intercepció de vol i de caiguda a la zona afectada durant un període d'un mes. Aquesta campanya va aportar un gran nombre d'interessants novetats per a la biodiversitat de la zona (Viñolas et al., 2013). L'any següent, a la vista dels resultats obtinguts el 2012, es va realitzar, a la mateixa àrea, un mostreig durant un període més llarg de temps (des de finals de maig fins a setembre), i amb una metodologia més completa amb relació al 2012. Es van instal-lar paranys d'intercepció de vol, de caiguda, de berlese in situ i de laboratori, i de llum ultraviolada, complementant aquests amb recol-leccions amb mànega i paraigua japonès, per així poder obtenir una millor visió de la biodiversitat de coleòpters existents al Tillar.

Dels exemplars estudiats s'ha de destacar la confirmació de la presència de Synchita undata Guérin-Méneville, 1884 (Zopheridae) a la península Ibèrica, zona en què havia estat citada amb dubtes. Es relacionen i es comenten 14 espècies de diferents famílies que bé o són noves per a Catalunya o n'hi ha escasses dades de l'àrea catalana. També s'adjunta una taula amb 19 espècies no citades fins ara de la província de Tarragona.

En el cas del Dermestidae Orphilus beali Zhantiev, 2001, se'n representa l'edeagus i de l'espècie veïna Orphilus niger (Rossi, 1790), capturades en la mateixa localitat i única manera segura de diferenciar-les, i se'n comenta la biologia.

S'ha seguit l'ordenació taxonòmica de Bouchard et al. (2011).

\section{Espècies confirmades per a la península Ibèrica}

Família Zopheridae Solier, 1834

Subfamília Colydiinae Billberg, 1820

Tribu Synchitini Erichson, 1845

Synchita undata Guérin-Méneville, 1844

Synchita undata Guérin-Méneville, 1844: 189

Cicones picta Erichson, 1845: 273

Material estudiat

2 ex., etiquetats: «la Maquina Vella, l'Albera, la Jonquera, Girona, 410 m, 25-VI2011, J. Muñoz \& J. Soler leg.» Amb parany d'intercepció de vol col·locat a la 
vora d'un torrent en una zona de bosc mixt de Quercus ilex L., Quercus pubescens Willd., Acer campestre L., Acer monspessulanum L. i amb molts exemplars morts d'Alnus glutinosa (L.) Gaertn. Per a més informació sobre la zona, vegeu Viñolas et al. (2012).

Després de la descripció de Sinchyta fallax Schuh, 1998, molt propera d'undata i coneguda a Espanya de Cadis (Viñolas \& Verdugo, 2011) i Navarra, s'han de revisar les citacions ibèriques de $S$. undata Guérin-Méneville, 1844, tal com indiquen Diéguez Fernández et al. (2012), ja que quedava dubtosa la seva presència a la Península. Amb la captura dels exemplars de 1'Albera (Girona), podem confirmar la presència de l'espècie a Espanya.

Sinchyta fallax i $S$. undata se separen bé per la diferent disposició de les setes elitrals així com per la diferent conformació dels paràmers de l'aparell genital.

\section{Espècies noves o poc conegudes per a Catalunya}

Família Eucnemidae Eschscholtz, 1829

Subfamília Melasinae Fleming, 1821

Tribu Dirhagini Reitter, 1911

Microrhagus emyi (Rouget, 1856)

Elater emyi Rouget, 1856: 193

\section{Material estudiat}

2 ô i 1 , , etiquetats: «la Maquina Vella, l'Albera, la Jonquera, Girona, $410 \mathrm{~m}$, 25-VI-2011, J. Muñoz \& J. Soler leg.» Amb parany d'intercepció de vol ubicat en el mateix lloc que s'indica per a l'espècie anterior.

Espècie ben diferenciada dels seus congèneres per la longitud dels apèndixs antenals, a part dels caràcters de la genitàlia i citada d'Espanya en el catàleg paleàrtic (Muona, 2007), tot i que Recalde Irurzun (2008) indica que l'única citació coneguda del país és la de Méquignon (1930), només amb la indicació «Espanya». Aquest autor, però, va obviar que posteriorment es va citar de Ponferrada (Lleó) (Horion, 1953). Així doncs, aquesta citació és la primera per a Catalunya.

Família Scarabaeidae Latreille, 1802

Subfamília Melolonthinae Leach, 1819

Tribu Sericini Kirby, 1837

Euserica segurana (Brenske, 1897)

Serica segurana Brenske, 1897: 364

\section{Material estudiat}

34 ex., etiquetats: 8 ex. «el Tillar, serra de Prades, Vimbodí i Poblet, Tarragona, 900 m, 31-V-2013, A. Viñolas leg.»; 16 ex. «el Tillar, serra de Prades, Vimbodí i Poblet, Tarragona, 900 m, 13-VI-2013, J. Muñoz»; 10 ex. «el Tillar, serra de Prades, Vimbodí i Poblet, Tarragona, 900 m, 18-VI-2013, A. Viñolas leg.» Tots amb paranys de llum ultraviolada. 
Endemisme espanyol conegut de nombroses províncies segons Murria Beltrán \& López-Colón (2002), citada d'Albacete, Alacant, Almerìa, Cadis, Conca, Ciudad Real, Granada, Guadalajara, Huelva, Jaén, Màlaga, Madrid, Múrcia, Osca, Terol, València i Saragossa. Espècie molt nombrosa al Tillar, ja que no es van recol-lectar tots els exemplars que van ser atrets pels paranys de llum ultraviolada, estant i tot present en els de caiguda. La citació de Tarragona és la primera per a Catalunya.

Família Buprestidae Leach, 1815

Subfamília Polycestinae Lacordaire, 1857

Tribu Acmaeoderini Kerremans, 1893

Acmaeodera (Palaeotethya) nigellata Abeille de Perrin, 1904

Acmaeodera nigellata Abeille de Perrin, 1904: 222

Acmaeodera satanula Reitter, 1907: 205

Acmaeodera lusitanica Obenberger, 1914: 251

Acmaeodera kureimatica Cobos, 1958: 232, 250

Material estudiat

2 ex., etiquetats: 1 ex. «el Tillar, serra de Prades, Vimbodí i Poblet, Tarragona, 900 m, 13-VI-2013, J. Muñoz»; 1 ex. «el Tillar, serra de Prades, Vimbodí i Poblet, Tarragona, 900 m, 27-VII-2013, A. Viñolas leg.».

Espècie amb poques i disperses citacions ibèriques (Verdugo, 2003). Arnáiz Ruiz et al. (2002) indiquen la seva presència en les províncies d'Àvila, Cáceres, Cadis, Lleó, Madrid, Osca, Ourense, Salamanca i Terol, a més de diverses localitats portugueses. Muñoz Batet et al. (2003), en l'ampliació de la corologia dels buprèstids ibèrics i de les Illes Balears, no aporten cap localitat nova per a l'espècie. Els exemplars del Tillar són la primera citació per a Catalunya.

Família Elateridae Leach, 1815

Subfamília Cardiophorinae Candèze, 1859

Cardiophorus (Cardiophorus) castillanus Buysson, 1902

Cardiophorus castillanus Buysson, 1902: 435

Cardiophorus arribasi Cobos, 1961: 75

\section{Material estudiat}

6 ex., etiquetats: «el Tillar, serra de Prades, Vimbodí i Poblet, Tarragona, 900 m, 13-VI-2013, J. Muñoz \& J. Soler leg.» Amb parany d'intercepció de vol.

Espècie coneguda d'Espanya, França, Itàlia i Portugal, amb una àmplia distribució ibèrica (Zapata de la Vega \& Sánchez-Ruiz, 2012). L'any següent amplien la distribució de l'espècie a la Península a les províncies de Cadis i de Lugo (Zapata de la Vega \& Sánchez-Ruiz, 2013), i recentment a la de Càceres i a l'Algarve, a Portugal. No indiquen la presència de l'espècie a Catalunya, i per això els exemplars del Tillar (Tarragona) són la primera citació documentada per a Catalunya. 
Família Dermestidae Latreille, 1804

Subfamília Orphilinae LeConte, 1861

Orphilus beali Zhantiev, 2001

Orphilus beali Zhantiev, 2001: 612

\section{Material estudiat}

4 ô i 2 + , etiquetats: «el Tillar, serra de Prades, Vimbodí i Poblet, Tarragona, 900 m, 12-VI / 4-VII-2013, A. Viñolas leg.» Amb mànega.

Espècie recentment descrita $\mathrm{i}$ amb molt poques citacions ibèriques. Coneguda d'Espanya, a Alacant, Barcelona i Jaén, i de Portugal, a Castelo Branco (Herrmann \& Baena, 2004; Diéguez Fernández, 2010; Háva, 2010). Els exemplars del Tillar són la primera citació de l'espècie per a Tarragona.

Al Tillar és una espècie simpàtrica amb Orphilus niger (Rossi, 1790), de la qual només es pot separar amb seguretat per la conformació de l'edeagus (Fig. 1-2), ja que externament són pràcticament indiferenciables. Aquesta qüestió fa que la majoria de les citacions d'aquesta espècie abans de la descripció d'O. beali hagin de ser confirmades. Els exemplars estudiats d'O. beali van ser capturats en les inflorescències d'una mata ruderal de Dauca carota L. i en les flors blanques de diverses espècies de Cistus sp., i situades en el camí d'entrada a la
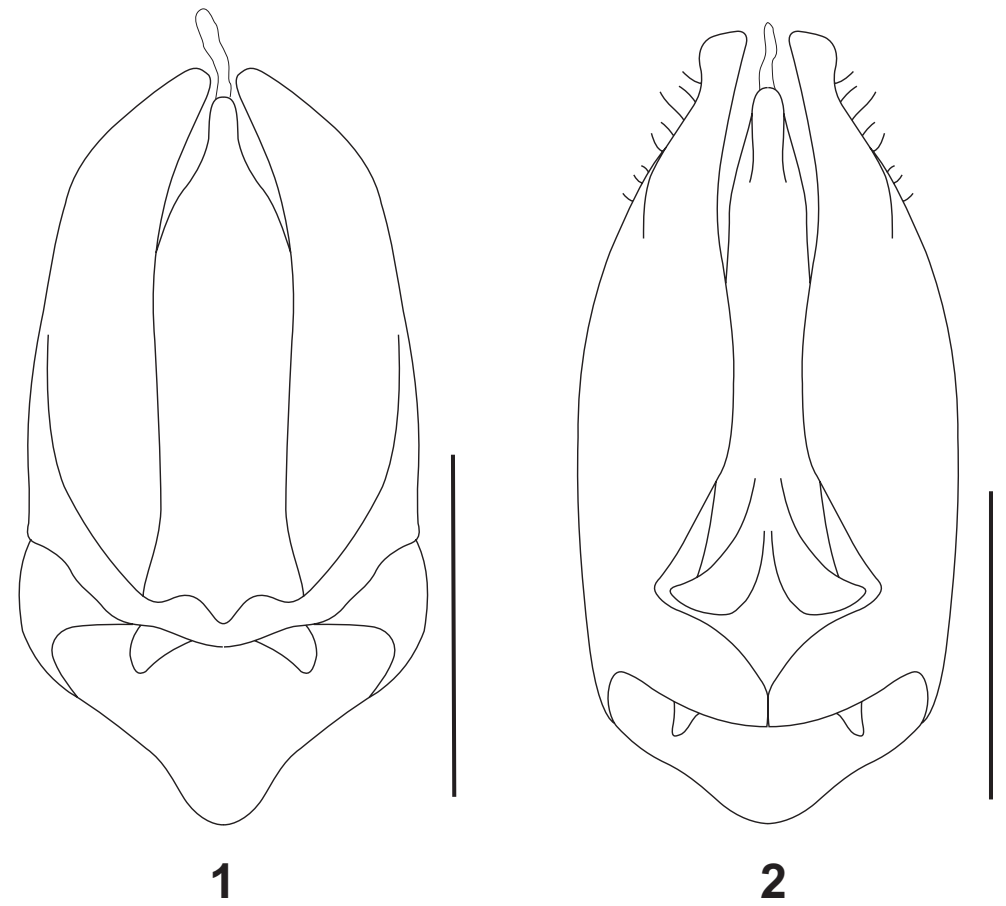

Figures 1-2. Edeagus en visió ventral de: 1. Orphilus niger; 2. O. beali. Escala =0,3 m. 
casa forestal del Tillar; ara bé, en les mateixes plantes i flors, a partir del 10 de juliol endavant només es va recol-lectar $O$. niger, no present abans d'aquesta data. Herrmann \& Baena (2004) també indiquen la presència d'O. beali en les flors de D. carota.

Representem els edeagus d'ambdues espècies per facilitar-ne la comparació.

Subfamília Trinodinae T.L. Casey, 1900

Tribu Trinodini T.L. Casey, 1900

Trinodes hirtus (Fabricius, 1781)

Nitidula hirta Fabricius, 1781: 93

Trinodes ater Dalla Torre, 1879: 100

Trinodes castanea Dalla Torre, 1879: 100

Trinodes testacea Dalla Torre, 1879: 100

Material estudiat

2 ex., etiquetats: «la Maquina Vella, l'Albera, la Jonquera, Girona, 410 m, 25-VI2011, J. Muñoz \& J. Soler leg.» Amb parany d'intercepció de vol.

Espècie coneguda de tot Europa fins al Caucas, i també d'Algèria i Tunísia. Les úniques citacions ibèriques que hem pogut localitzar són la de Barcelona (Horion, 1955) i la de Terol (Háva et al., 2010); la present és, doncs, la segona citació per a Catalunya.

Segons Háva \& Nardi (2004), les larves d'aquesta espècie es troben al costat de les teles d'aranya, sota escorça, en fusta en descomposició, soques i buits d'arbres, on s'alimenten d'artròpodes morts o de les seves restes.

Família Ptinidae Latreille, 1802

Subfamília Ernobiinae Pic, 1912

Ochina ptinoides (Marsham, 1802)

Crioceris ptinoides Marsham, 1802: 228

Birrhus fasciatus Geoffroy, 1785: 26

Ptilinus hederae P.W.J. Müller, 1821: 193

Material estudiat

2 ex., etiquetats: «la Maquina Vella, l'Albera, la Jonquera, Girona, 410 m, 25-VI2011, J. Muñoz \& J. Soler leg.» Amb parany d'intercepció de vol.

Espècie que es coneixia de la Península per només una citació de Portugal (Español, 1992), fins que Baselga \& Novoa (2000) van citar una femella d'A Coruña (Guiliade-Betazos) i posteriorment Bercedo \& Arnáiz (2005) citen un mascle de Madrid (Torres de la Alameda). Els exemplars de Girona són la primera citació catalana i del mediterrani ibèric, ampliant notablement la distribució peninsular de l'espècie.

Segons Español (1992), l'espècie es desenvolupa en branques d'heura. Bercedo \& Arnáiz (2005) indiquen que l'exemplar de Madrid es va obtenir per emergència d'una branca de Colutea arborescens. Dels exemplars de Galícia i de 
Catalunya no es tenen dades sobre la seva biologia, pel fet de ser capturats amb diferents models de paranys.

Família Bothrideridae Erichson, 1845

Subfamília Teredinae Seidlitz, 1888

Teredus cylindricus (A.G. Olivier, 1790)

Ips cylindricus A.G. Olivier, 1790: no 18: 9

Lyctus nitidus Fabricius, 1792: 505

Material estudiat

1 ex., etiquetat: «Còrrec de la Vernosa, l'Albera, la Jonquera, Girona, 22-VII2013, J. Muñoz». Sota l'escorça d'un Acer campestris L., abatut.

Espècie citada de Navarra per Recalde Irurzun \& San Martín Moreno (2007), i tal com indiquen, encara que està citada de Portugal i d'Espanya en el catàleg paleàrtic, no hem localitzat cap altra menció ibèrica de l'espècie. Creiem que l'exemplar de Girona és la primera per a Catalunya.

Segons Recalde Irurzun \& San Martín Moreno (2007), es localitza sota l'escorça de vells roures, on depreda larves d'anòbids, escolítids i cerambícids.

Família Mycetophagidae Leach, 1815

Subfamília Mycetophaginae Leach, 1815

Triphyllus bicolor (Fabricius, 1777)

Nitidula bicolor Fabricius, 1777: 216

Mycetophagus punctatus Fabricius 1792b: 499

Kryptophagus pilosus Herbst, 1792: 177

Silpha humeralis Marsham, 1802: 123

Tripyllus immaculatus Roubal, 1936a: 192

\section{Material estudiat}

1 ex., etiquetat: «el Tillar, serra de Prades, Vimbodí i Poblet, Tarragona, 900 m, 2-VII-2013, J. Muñoz \& J. Soler leg.» Amb parany d'intercepció de vol en un bosc mixt de Quercus sp. (alzines i roures) i Pinus sylvestris L.. Per a més informació sobre la zona mostrejada, vegeu Viñolas et al., 2013.

Espècie micetòfaga present en la «European Red List of Saproxylic Beetles» (Nieto \& Alexander, 2010) amb la categoria UICN «LC». Poques són les localitzacions ibèriques conegudes. Pagola Carte (2007) la cita de Gipuzkoa i La Rioja, també indiquen la seva presència a Portugal, i Recalde Irurzun \& San Martín Moreno (2010) amplien la seva àrea a Navarra. Creiem que l'exemplar de Tarragona és la primera cita per a Catalunya.

Segons Pagola Carte (2007), es desenvolupa en fongs lignícoles de caducifolis, és citat de Fistula hepatica (Schaeff.) With. (1792) i de Laetiporus sulphureus (Bull.) Murrill (1920). 
Família Scraptiidae Gistel, 1848

Subfamília Anaspidinae Mulsant, 1856

Anaspis garneysi Fowler, 1889

Anaspis garneysi Fowler, 1889: 333

Material estudiat

16 ex., etiquetats: 3 ex. «turó de Can Balasc, Collserola, Barcelona, 19-V-2012, A. Viñolas leg.»; 1 ex. «turó de Can Balasc, Collserola, Barcelona, 1/31-V-2012, J. Mederos leg.»; 12 ex. «el Tillar, serra de Prades, Vimbodí i Poblet, Tarragona, 900 m, 16/25-VI-2012, F. Benvenuti \& A. Viñolas leg.» Amb parany d'intercepció de vol en la mateixa zona que l'espècie anterior.

Espècie amb poques citacions europees (Alemanya, Països Baixos i Regne Unit), l'única de la Península és la del Parc Natural de la serra Cebollera (La Rioja) (Pérez-Moreno \& Moreno Grijalba, 2007). Els exemplars del Tillar (Tarragona) i els de Collserola (Barcelona) són les primeres per a Catalunya.

La larva és xilòfaga i es desenvolupa en la fusta de frondoses.

Cyrtanaspis phalerata (Germar, 1847)

Anaspis phalerata Germar, 1847: 8

Cyrtanaspis obscura Schilsky, 1895: 60

Cyrtanaspis interrupta Schilsky, 1908: 606

\section{Material estudiat}

4 ex., etiquetats: 1 ex. «el Tillar, serra de Prades, Vimbodí i Poblet, Tarragona, 900 m, 16/25-VI-2012, F. Benvenuti \& A. Viñolas leg.» Amb parany d'intercepció de vol; 3 ex. «el Tillar, serra de Prades, Vimbodí i Poblet, Tarragona, 900 m, 2-VII-2013, J. Muñoz \& J. Soler leg.» Amb parany d'intercepció de vol.

Dues són les citacions ibèriques conegudes de l'espècie, una de La Rioja (Pérez Moreno \& Moreno Grijalba, 2007) i l'altra de Barcelona (Viñolas et al., 2012), a les quals podem afegir la del Tillar, serra de Prades, Tarragona.

L'espècie es desenvolupa en la fusta en descomposició de diferents frondoses. Tots els exemplars capturats a la Península ho han estat en boscos mixtos de Quercus sp. y Pinus sp.

Família Cerambycidae Latreille, 1802

Subfamília Cerambycinae Latreille, 1802

Tribu Hesperophanini Mulsant, 1839

Subtribu Hesperophanina Mulsant, 1839

Trichoferus magnanii Sama, 1992

Trichoferus magnanii Sama, 1992: 398

\section{Material estudiat}

1 ふै, etiquetat: «el Tillar, serra de Prades, Vimbodí i Poblet, Tarragona, 900 m,

5-VII-2013, A. Viñolas leg.» Amb parany de llum ultraviolada. 
Espècie amb unes quantes citacions ibèriques, amb una única citació de cadascuna de les províncies següents, excepte de Madrid, amb dues: Cadis, Conca, Còrdova, Jaén, Madrid, Múrcia, Saragossa i Sevilla (Sama, 1992; Murria et al., 1997; Vives \& Sama, 1998; Vives, 2001; Verdugo \& Coello, 2003; Verdugo Páez, 2004; Navarro et al., 205; Veronese, 2005; de la Rosa, 2005), i és la del Tillar la primera per a Catalunya.

La larva es desenvolupa, segons Sama (1992), en Cistus creticus L. Vives (2001) indica a $C$. villosus L., sinònim de $C$. creticus, espècie que no es coneix de moltes de les localitats citades, i no present a Catalunya. Verdugo \& Coello (2003) diuen que es desenvolupa en els ceps i les arrels de $C$. ladanifer L. L'exemplar del Tillar va ser capturat a la llum en una zona amb presència de $C$. ladanifer.

Tribu Molorchini Gistel, 1848

Molorchus (Molorchus) marmottani marmottani (Brisout de Barneville, 1863) Glaphyra marmottani Brisout de Barneville, 1863: 118

Molorchus discicollis Heyden, 1876: 383

\section{Material estudiat}

2 ex., etiquetats: $1 \hat{\jmath}$ «el Tillar, serra de Prades, Vimbodí i Poblet, Tarragona, 900 m, 20-VII-2013, A. Viñolas leg.» Amb parany de llum ultraviolada; 1 ๙ «el Tillar, serra de Prades, Vimbodí i Poblet, Tarragona, 900 m, 20-VII-2013, J. Muñoz \& J. Soler leg.» Amb parany d'intercepció de vol.

Espècie només coneguda a la península Ibèrica de Cadis, Granada, Lleida, Madrid, Osca i Terol (González et al., 2003). De Catalunya només es coneixia de Montellà i Martinet (Lleida); els exemplars del Tillar són la segona citació per a Catalunya i la primera per a Tarragona.

Segons Vives (2001), la larva es desenvolupa en les branques seques de diferents espècies de Pinus. L'única espècie de pi present a l'àrea del Tillar és el pi roig ( $P$. sylvestris).

Família Curculionidae Latreille, 1802

Subfamília Baridinae Schönherr, 1836

Tribu Baridini Schönherr, 1836

Aulacobaris coerulescens coerulescens (Scopoli, 1763)

Curculio coerulescens Scopoli, 1763: 29

Curculio chalybeus Gmelin, 1790: 1759

\section{Material estudiat}

1 ex., etiquetat: «Can Costoja, Rabós, Girona, 5-IV-2009, J. Soler leg.».

D'aquesta espècie només hem trobat les citacions de la comunitat de Madrid (Alonso-Zarazaga et al., 2006) i d'Araba (Parque Natural de Valderejo) (Ugarte San Vicente \& Salgueira Cerezo. 2006). No tenim constància de cap citació prèvia de l'espècie de Catalunya.

Espècie fitòfaga que es desenvolupa en diferents espècies de Brassicaceae, 
citada de Brassica oleracea L., B. rapa L. subsp. oleifera DC. i de Matthiola fruticulosa (L.) Maire in Jahand. et Maire subsp. fruticulosa. L'adult es pot localitzar en crucíferes tals com Barbarea vulgaris R. Br. in Aiton, Diplotaxis erucoides (L.) DC., Diplotaxis tenuifolia (L.) DC. i Sinapis arvensis L., entre altres.

Subfamília Mesoptiliinae Lacordaire, 1863

Tribu Magdalidini Pascoe, 1870

Magdalis (Magdalis) duplicata Germar, 1819

Magdalis duplicata Germar, 1819: 131

Magdalinus punctipennis Küster, 1851: 84

Magdalinus parallelocollis Desbrochers des Longes, 1870: 31

Magdalis weisei Schreiner, 1882: 159

Magdalis formaneki Reitter, 1895: 302

Material estudiat

1 ex., etiquetat: «Requesens, la Jonquera, Girona, 19-V-1997, J. Soler leg.».

També d'aquesta espècie només hem trobat les citacions de la comunitat de Madrid (Alonso-Zarazaga et al., 2006) i d'Araba (Parque Natural de Valderejo) (Ugarte San Vicente \& Salgueira Cerezo. 2006). No tenim constància de cap citació prèvia de l'espècie de Catalunya.

Espècie fitòfaga, com l'anterior, que completa el seu cicle biològic en branques de resinoses. S'ha citat de Picea excelsa (Lam.) Link, Pinus sylvestris, $P$. uncinata Raymond ex A. DC. i Larix decidua Mill., 1768.

\section{Espècies citades per primera vegada de Tarragona}

En la taula 1 es relacionen, agrupades per famílies i alfabèticament, les espècies que se citen per primera vegada de la província de Tarragona. L'apartat «referència» indica la bibliografia de les diferents revisions de cada grup, on no es preveu la presència d'aquestes a la província. 1. Arnáiz Ruiz et al., 2002; Muñoz Batet et al., 2003; Verdugo, 2003. 2. González Peña et al., 2007; 3. Petitpierre, 2009; 4. Zapata de la Vega \& Sánchez-Ruiz, 2012, 2013, 2014; 5. Español, 1956; Viñolas et al., 2012; Diéguez Fernández, 2013.

\section{Agraïments}

Agraïm a Bertomeu Borràs, director del Paratge d'Interès Nacional de l'Albera, les facilitats ofertes per poder realitzar l'estudi de biodiversitat durant aquests anys. A Xavier Buqueras, director del Paratge Natural d'Interès Nacional de Poblet, les facilitats ofertes per a l'estudi de la biodiversitat de coleòpters en l'àrea de la casa forestal del Tillar. A Lluís Cabañeros i a Francesc Llimona la seva ajuda i col-laboració en l'estudi de la biodiversitat de coleòpters al Parc Natural de la serra de Collserola.

A Maurizio Mencuccini, del Centre de Recerca Ecològica i Aplicacions Forestals (CREAF), de Bellaterra, la col·laboració i ajut en les recol·leccions efectuades 
Taula 1. Espècies recol-lectades al Tillar, serra de Prades, i no citades de Tarragona

\begin{tabular}{|c|c|c|}
\hline Família & Espècie & Referència \\
\hline \multirow[t]{12}{*}{ Buprestidae } & Agrilus (Agrilus) cuprescens cuprescens (Ménétriés, 1832) & 1 \\
\hline & Agrilus (Agrilus) viridis viridis Linnaeus, 1758 & 1 \\
\hline & Agrilus (Anambus) biguttatus (Fabricius, 1777) & 1 \\
\hline & $\begin{array}{l}\text { Agrilus (Quercuagrilus) angustulus angustulus (Illiger, } \\
\text { 1803) }\end{array}$ & 1 \\
\hline & $\begin{array}{l}\text { Agrilus (Quercuagrilus) graminis graminis Kiesenwetter, } \\
1857\end{array}$ & 1 \\
\hline & Agrilus (Quercuagrilus) laticornis (Illiger, 1803) & 1 \\
\hline & $\begin{array}{l}\text { Agrilus (Xeragrilus) albogularis artemisiae Brisout de } \\
\text { Barneville, } 1863\end{array}$ & 1 \\
\hline & $\begin{array}{l}\text { Anthaxia (Haplanthaxia) ignipennis Abeille de Perrin, } \\
1882\end{array}$ & 1 \\
\hline & $\begin{array}{l}\text { Anthaxia (Haplanthaxia) scutellaris scutellaris Gené, } \\
1839\end{array}$ & 1 \\
\hline & $\begin{array}{l}\text { Buprestis (Ancylocheira) haemorrhoidalis } \\
\text { haemorrhoidalis Herbst, } 1790\end{array}$ & 1 \\
\hline & Meliboeus (Meliboeus) fulgidicollis (P.H. Lucas, 1846) & 1 \\
\hline & Meliboeus (Meliboeus) graminis graminis (Panzer, 1799) & 1 \\
\hline \multirow[t]{2}{*}{ Cerambycidae } & Stenurella bifasciata bifasciata (O.F. Müller, 1776) & 2 \\
\hline & Trichoferus pallidus (Olivier, 1790) & 2 \\
\hline Chrysomelidae & Sermylassa halensis (Linnaeus, 1767) & 3 \\
\hline \multirow[t]{3}{*}{ Elateridae } & Athous (Athous) puncticollis Kiesenwetter, 1858 & 4 \\
\hline & Pleurathous godarti (Mulsant \& Guillebeau, 1856) & 4 \\
\hline & Stenagostus laufferi (Reitter, 1904) & 4 \\
\hline Erotylidae & Triplax melanocephala (Latreille, 1804) & 5 \\
\hline
\end{tabular}

al Tillar, Tarragona, l'any 2012. A Francesco Benvenuti, d'Itàlia; a Ramon Macià, de Vic, i a Josep Ylla, de Gurb, la col-laboració en els mostreigs efectuats al Tillar els anys 2012 i 2013. A Jorge Mederos, de Barcelona, l'haver-nos permès estudiar els coleòpters recol-lectats, per ell, al turó de Can Balasc, Collserola.

A José Carlos Otero, de la Universitat de Santiago de Compostel-la pel seu ajut en la recerca de bibliografia.

\section{Referències bibliogràfiques}

Abeille de Perrin, E. 1904. Buprestides. Boletín de la Real Sociedad Española de Historia Natural, 4: 206-224.

Alonso-Zarazaga, M.A.; Sánchez-Ruiz, M.; Domingo-Quero, T. 2006. Lista preliminar de los Curculionoidea de la Comunidad de Madrid (España). Graellsia (número extraordinario), 62: 43-52. 
Arnáiz Ruiz, L.; Bercedo Páramo, P.; Zuzarte, A.J. de Sousa. 2002. Corología de los Buprestidae de la Península Ibérica e Islas Baleares (Coleoptera). Boletín de la Sociedad Entomológica Aragonesa, 30: 37-80.

Baselga, A. \& Novoa, F. (2000). Nuevas citas de Anobiidae (Coleoptera) de Galicia (Península Ibérica). Boletín de la Asociación Española de Entomología, 24 (3-4): 211212.

Bercedo, P.; Arnáiz, L. 2005. Apuntes sobre anóbidos ibéricos I: dos carcomas poco conocidas de España (Coleoptera: Anobiidae). Boletín de la Sociedad Entomológica Aragonesa, 37: 140.

Brenske, E. 1897. Die Serica-Arten der Erde. Monographisch bearbeitet. Berliner Entomologische Zeitscrift, 42: 345-438.

Brisout de Barneville, C.N.F. 1863. [New taxa]. In: Grenier, A.: Catalogue des coléoptères de France par M. le Dr. A. Grenier et matériaux pour servir à la faune des coléoptères français. A. Grenier. Paris. iv + 3-79 + 1-135 p.

Bouchard, P.; Bousquet, Y.; Davies, A.E.; Alonso-Zarazaga, M.A.; Lawrence, J.F.; Lyal, C.H.C.; Newton, A.F.; Reid, C.A.M.; Schmitt, M.; Ślipiński, S.A.; Smith, A.B.T. 20 I I. Family-group names in Coleoptera (Insecta). ZooKeys, 88: I-972.

Buysson, H. du. 1902. Remarques sur quelques életérides du genre Cardiophorus et descriptions d'espèces nouvelles. Annales de la Société Entomologique de France, 71 (1902-1903): 422-439.

Cobos, A. 1958. Revisión de los Acmaeoderini de Marruecos (Col. Buprestidae). EOS, Revista Española de Entomología, 34: 221-268.

Cobos, A. 1961. Exploración entomológica del cedral de Tizi-Ifri (macizo del Iguelmalet, Rif central, Marruecos). Coleópteros. Archivos del Instituto de Aclimatación, Almería, 10: 63-98, + 1 pl.

Dalla Torre, K.W. von. 1879. Die Käferfauna von Oberösterreich. Systematisches Verzeichnis der in Oberösterreich bisher beobachteten Käfer. Jahresberich des Vereins für Naturkunde in Oesterreich ob der Enns zu Linz, 10: 1-125.

Desbrochers des Loges, J. 1870. Monographie des Magdalinus d'Europe et des pays circum-méditerranéens. L'Abeille, Memoires d'Entomologie, 7: 1-61.

Diéguez Fernández, J.M. 2010. Nuevos coleópteros para Cataluña (NE Península Ibérica) (Insecta: Coleoptera). Arquivos Entomolóxicos, 3: 87-89.

Diéguez Fernández, J.M. 2013. Registros interesantes de coleópteros para España (Insecta Coleoptera). $2^{\mathrm{a}}$ nota. Arquivos Entomolóxicos, 8: 277-286.

Diéguez Fernández, J.M.; Recalde Irurzun, J.I.; Schuh, R. 2012. Aportaciones a la corología de los Zopheridae ibéricos. Heteropterus Revista de Entomología, 12 (1): 107-114.

Español, F. 1956. Los erotílidos (Col.) del macizo del Montseny (Barcelona). Graellsia, 14: $1-12$.

Español, F., 1992. Coleoptera Anobiidae. In: Fauna Ibérica, vol. 2. Ramos, M.A. et al. (Eds.). Museuo Nacional de Ciencias Naturales, CSIC. Madrid. 195 p.

Erichson, W.F. 1845. Lieferung II. P. 161-320. In: Naturgeschichte der Insecten Deutschlands. Erste Abtheilung. Coleoptera. Dritter Band. Nicolaischen Buchhandlung. Berlin. $320 \mathrm{p}$.

Fabricius, J.C. 1777. Genera insectorum eorumque characteres naturales secundum numerum, figuram, situm et proportionem omnium partium oris adiecta mantissa specierum nuper detectarum. Chilonii: Mich. Friedr. Bartschii, $14+310$ p.

Fabricius, J.C. 1781. Species insectorum exhibentes eorum differentias specificas, synonyma auctorum, loca natalia, metamorphosis adiectis observationibus, descriptionibus. Tom I. C.E. Bohn. Hamburgi et Kilonii. viii +552 p. 
Fabricius, J.C. 1792. Entomologia systematica emendata et aucta. Secundum classes, ordines, genera, species adjectis synonymis, locis, descriptionibus, observationibus. Tom I. Pars II. Christ. Gottl. Proft. Hafiniae. 538 p.

Fowler, M.A. 1889. On the British species of the genus Anaspis, Geoffroy, with description of a new species. The Entomologist's Monthly Magazine, 25: 331-336.

Germar, E.F. 1819. Versuch einer Eintheilung der Horde der Rüsselkäfer in mehrere Gattungen. Annalen der Wetterauischen Gesellscharf für dir Gesammte Naturkunde, 4(1) (1818): 116-139.

Germar, E.F. 1847. Fauna Insectorum Europae. Fasciculus 24. Halae: $[2+25]$ p. +25 pl.

Geoffroy, E.L. 1785. [new taxa]. In: Fourcroy, A.F. de: Entomologia parisiensis; sive Catalogus Insectorum quae in Agro Parisiensi reperiuntur; Secundum methodum Geoffraeanam in sectiones, genera et species distributus; cui addita sunt nomina trivialia et fere trecentae novae Specis. Pars prima. Privilegio Academinae. Parisiis. vii $+[1]+$ $231 \mathrm{p}$.

Gmelin, J.F. 1790. Caroli a Linné, Systema Naturae per regna tria naturae, secundum classes, ordines, genera, species, cum characteribus, differentis, synonymis, locis. Editio decima tertia, aucta, reformata. Tom. I. Pars IV. Classis V. Insecta. Georg Emanuel Beer. Lipsiae. P. 1517-2224.

González Peña, C.F.; Vives Noguera, E.; Zuzarte, A.J. de Sousa. 2007. Nuevo catálogo de los Cerambycidae (Coleoptera) de la Península Ibérica, islas Baleares e islas atlánticas: Canarias, Açores y Madeira. Monografías de la Sociedad Entomológica Aragonesa, 12: $1-211$.

Guérin-Méneville, F.E. 1844. Iconographie du Règne Animal de G. Cuvier, ou représentation d'après nature de l'une des espèces les plus remarquables, et souvent non encore figurées, de chaque génère d'animaux. Avec un texte descriptif mis au courant de la science, Ouvrage pouvant servir d'atlas à tous les traités de zoologie. III. Texte explicatif. Insectes. J.B. Baillière. Paris. 576 p.

Háva, J. 2007. Dermestidae. P. 57, 299-320. In: Löbl, I \& Smetana, A. (Eds.). Catalogue of Palaearctic Coleoptera, vol. 4. Elateroidea, Derodontoidea, Bostrichoidea, Lymexyloidea, Cleroidea and Cucujoidea. Apollo Books. Stenstrup. 935 p.

Háva, J. 2010. Nuevos datos sobre Orphilus beali Zhantiev para la Península Ibérica (Coleoptera: Dermestidae). Arquivos Entomolóxicos, 3: 99-100.

Háva, J.; Herrmann, A.; Plata Negrache, P. 2010. Catálogo de los Dermestidae (Coleoptera) de Aragón. Catalogus entomofauna aragonesa, 35: 69-73.

Háva, J. \& Nardi, G. 2004. Preliminary data on the Nosodendridae and Dermestidae (Coleoptera). Invertenrati di una foresta della Pianura Padana Bosco della Fontana, Secondo contributo - Conservazione Habitat Invertebrati, 3: 117-123.

Herbst, J.F.W. 1792. Natursystem aller bekannten in- und ausländischen Insekten, als eine Fortsetzung der von Buffonschen Naturgeschichte. Der Käfer vierter Theil. Mit zwölf illuminierten Kupferstafeln. Berlin : Joachim Pauli, viii +197 p. +12 pls.

Herrmann, A.; Baena, M. 2004. New records of Dermestidae (Coleoptera) for Spain and Europe. Boletín de la Sociedad Entomológica Aragonesa, 34: 211-213.

Heyden, L.F.J.D. von 1876. Molorchus discicollis Heyd. Deutsche Entomologische Zeitschrift, 20: 383-384.

Horion, A. 1953. Faunistik der Mitteleuropäischen Käfer, Bd. III, Malacodermata, Sternoxia (Elateridae - Throscidae). Eigenverlag Museum G. Frey, München. 340 p.

Horion, A. 1955. Faunistik der Mitteleuropäischen Käfer, Bd. IV, Sternoxia (Buprestidae), Fossipedes, Macrodactylia, Brachymera. Eigenverlag Museum G. Frey, Tutzing bei München. 280 p. 
Küster, H.C. 1851. Die Käfer Europa's. Nach der Natur beschrieben. Mit Beiträgen mehrerer Entomologen. 23 Heft. Bauer und Raspe. Nürnberg. [4] p + 100nrs + 2 pl.

Marsham, T. 1802. Entomologia Britannica, sistens insecta britanniae indigena, secundum methodum linnaeanam disposita. Tomus I. Coleoptera. Wilks et Taylor, J. White. Londini. xxxi $+548 \mathrm{p}$.

Méquignon, A. 1930. Serricornia. In: Bedel, L. Faune des coléoptères du bassin de la Seine, Vol. 4 (3). Société Entomologique de France. Paris. 362 p.

Müller, P.W.J. 1821. Neue Insecten. Magazin der Entomologie, 4: 184-230.

Muñoz Batet, J.; Blasco Zumeta, J.; Viñolas, A. 2003. Nuevas aportaciones a la corología de los bupréstidos ibéricos y de las Islas Baleares (Coleoptera: Buprestidae). Boletín de la Sociedad Entomológica Aragonesa, 32: 161-167.

Muona, J. 2007. Eucnemidae. P. 81-87. In: Catalogue of Palaearctic Coleoptera. Volume 4. Elateroidea -. Derodontoidea - Bostrichoidea - Lymexyloidea - Cleroidea - Cucujoidea. Apollo Books. Stenstrup. 935 p.

Murria, F.; Murria, A.; Beltrán, J.R. 1997. Opsilia malachitica (Lucas, 1849) y Trichoferus magnanii Sama, 1992: dos nuevas especies de Cerambycidae para la Comunidad de Aragón. Boletín de la Sociedad Entomológica Aragonesa, 17: 62.

Murria Beltrán, F.; López-Colón, J.I. 2002. Nuevos registros de Euserica segurana para Aragón (Coleoptera Scarabaeidae, Melolonthinae, Sericinae). Boletín de la Sociedad Entomológica Aragonesa, 31: 201.

Navarro, J; Urbano, J.M.; Llinares, A. 2005. Primera cita de Trichoferus magnanii Sama, 1992 (Coleoptera, Cerambycidae) para Sevilla (Andalucía, España). Boletín de la Sociedad Entomológica Aragonesa, 37: 302

Nieto, A.; Alexander, K.N.A. 2010. European Red List of Saproxylic Beetles. Luxembourg: Publications Office of the European Union. 45 p.

Obenberger, J. 1914. Neue Acmaeoderen (Coleoptera-Buprestidae). Entomologische Blätter, 10: 250-254.

Olivier, A.G. 1790. Entomologie, ou histoire naturelle des insectes, avec leurs caractères génériques et spécifiques, leur description, leur synonymie, et leur figure enluminée. Coléoptères. Tome second. de Baudouin. Paris. 485 p. +63 pl.

Pagola Carte, S. 2007. Inventario y seguimiento de la entomofauna del hayedo de Oieleku (Oiartzun, Parque Natural de Aiako Harria) - Campaña 2007. Asociación Gipuzkoana de Entomología. 100 p.

Pérez Moreno, I.; Moreno Grijalba, F. 2007. Seis nuevos coleópteros (Coleoptera) para la fauna de la Península Ibérica. Boletín de la Sociedad Entomológica Aragonesa, 41: 335-336.

Petitpierre, E. 2009. Catàleg dels coleòpters crisomèlids de Catalunya V. Hispinae i Cassidinae, i llista actualitzada de totes les espècies de la família. Butlletí de la Institució Catalana d'Història Natural, 75 (2007-2009): 61-83.

Recalde Irurzun, J.I. 2008. Elementos para el conocimiento de los eucnémidos del norte de España y actualización del catálogo de especies ibéricas (Coleoptera: Elateroidea: Eucnemidae). Heteropterus Revista de Entomología, 8 (2): 233-252.

Recalde Irurzun, J.I.; San Martín Moreno, A.F. 2007. Presencia de Oxylaemus variolosus en la Península Ibérica y otras aportaciones sobre Teredinae de Navarra (Coleoptera: Cucujoidea: Bothrideridae). Heteropterus Revista de Entomología, 7 (1): 57-60.

Recalde Irurzun, J.I.; San Martín Moreno, A.F. 2010. Tenebrionoidea y Cucujoidea (Coleoptera) de los hongos lignícolas, nuevos o poco conocidos para la fauna ibérica. Heteropterus Revista de Entomología, 10 (2): 145-156. 
Reitter, E. 1895. Uebersicht der mir bekannten Arten der Coleopteren-Gattung Magdalis Germ. Deutsche Entomologische Zeitschrift: 297-302.

Reitter, E. 1907. Eine neue spanische Acmaeodera. Boletín de la Real Sociedad Española de Historia Natural, 7: 205.

Rosa, J. J. de la, 2005. Aportaciones al conocimiento de la fauna de cerambícidos de la Comunidad de Madrid y territorios limítrofes (II) (Coleoptera: Cerambycidae). Boletín de la Sociedad Entomológica Aragonesa, 36: 355-356.

Rossi, P. 1790. Fauna Etrusca, sistens Insecta, quae in provinciis Florentina et Pisana praesertim collegit. Tomus primus. Liburni: Thomae Masi \& Sociorum, xxiii +272 p.

Roubal, J. 1936. Katalog coleopter (brouků) Slovenska a Podkarpatské Rust na základé bionomickém a zoogeografickém a spolu systematický doplněk Ganglbauerových "Die Käfer von Mittleuropa" a Reitterovy "Fauna Germanica” Dil II. Bratislava: Učená spoleěnost Šafařikova v Bratislavě, $434 \mathrm{p}$.

Rouget, A. 1856. Catalogue des insectes coléoptères du Départment de la Côte-d'Or. Mémoires de l'Académie Impériale des Sciences, Arts et Belles-lettres de Dijon, 2 (4): 113-195.

Sama, G. 1992. Note sur les longicornes de la Péninsula Ibérique avec description d'une nouvelle espèce de Trichoferus. Biocosme Mésgéen, 8 (4), 9 (1): 395-400.

Schilsky, J. 1895. Die Käfer Europa's. Nach der Natur Beschrieben von Dr. H.C. Küster und Dr. G. Kraatz Fortgesetzt von J. Schilsky. Bauer und Raspe (Emil Küster). Nürnberg, 31: viii + no. taxa 1-100 + [1] p.

Schilsky, J. 1908. XII. Beitrag zur deutschen Käferfauna. Deutsche Entomologische Zeitscrift: $604-608$.

Schreiner, J.F. 1882. Magdalis Weisei Schreiner, neue deutsche Art. Deutsche Entomologische Zeitschrift, 26: 159-160.

Schuh, R. 1998. Revision of the Synchita variegata species group (Coleoptera: Zopheridae, Colydiinae). Annales Zoologici (Warszawa), 48 (3-4): 313-324.

Scopoli, I.A. 1763. Entomologia Carniolica; exhibens insecta Carnioliae indigena et distributa in ordines, genera, species, varietates; methodo linnaeana.I.T. Trattner. Vindobonae. xxxiv $+420 \mathrm{p}$.

Ugarte San Vicente, I.; Salgueira Cerezo, F. 2006. Los escarabajos (Insecta: Coleoptera) del Parque Natural de Valderejo, Araba (País Vasco). 1. parte. Amalur Natura Elkartea. Departamento de Medio Ambiente y Ordenación del Territorio (Gobierno Vasco). $212 \mathrm{p}$.

Verdugo Páez, A. 2003. Contribución a un mejor conocimiento de los bupréstidos ibéricos (Coleoptera, Buprestidae). Boletín de la Sociedad Entomológica Aragonesa, 32: 197200

Verdugo Páez, A. 2004. Los cerambícidos de Andaucía (Coleoptera: Cerambycidae). Monográfico ${ }^{\circ} 1$. Sociedad Andaluza de Entomología, Córdoba. 148 p.

Verdugo, A.; Coello, P. 2003. Contribución al conocimiento de los cerambícidos de Andalucía III. Phymatodellus rufipes (Fabricius, 1777); nueva especie andaluza y datos interesantes para otras seis (Coleoptera, Cerambycidae). Boletín de la Sociedad Entomológica Aragonesa, 32: 105-107.

Veronese, P. 2005. Primera cita de Trichoferus magnanii Sama, 1992 para la Comunidad Autónoma de Madrid (Coleoptera, Cerambycidae). Boletín Sociedad Entomológica Aragonesa, $36: 250$.

Viñolas, A.; Soler, J.; Muñoz-Batet, J. 2012. Nuevos registros y nuevas localizaciones de coleópteros para la Península Ibérica y en especial del Paratge natural de l'Albera, Girona (Coleoptera). Elytron, 25: 3-63. 
Viñolas, A.; Muñoz, J.; Mencuccini, M.; Benvenuti, F. 2013. Nuevos datos sobre Rushia parreyssi (Mulsant, 1856), Melandrydae Leach, 1815 y otros coleópteros interesantes de la sierra de Prades, Tarragona (Coleoptera). Orsis 27: 29-51

Viñolas, A.; Verdugo, A. 2011. Nuevas especies de coleópteros para la Península Ibérica. Familias Zopheridae, Corylophidae y Curculionidae. Orsis, 25: 131-139.

Vives, E. 2001. Atlas fotográfico de los cerambícidos íbero-baleares (Coleoptera). Argania editio. Barcelona. 287 p.

Vives, E.; Sama, G. 1998. Cerambycidae nouveaux ou peu connus de la faune d'Espagne (Coleoptera, Cerambycidae). Biocosme Mésogéen, 14 (4): 129-134.

Zapata de la Vega, J.L.; Sánchez-Ruiz, A. 2012. Catálogo actualizado de los Elatéridos de la Península Ibérica e Islas Baleares (Coleoprtera: Elateridae). Arquivos Entomolóxicos, 6: 115-271.

Zapata de la Vega, J.L.; Sánchez-Ruiz, A. 2013. Nuevas aportaciones al catálogo de la familia Elateridae (Coleoptera) en la Península Ibérica e Islas Baleares, I. Arquivos Entomolóxicos, 8: 159-190.

Zapata de la Vega, J.L.; Sánchez-Ruiz, A. 2014. Nuevas aportaciones al catálogo de la familia Elateridae (Coleoptera) en la Península Ibérica e Islas Baleares, II. Arquivos Entomolóxicos, 10: 129-166.

Zhantiev, R.D. 2001. Zhuki-kozheedy roda Orphilus Er. (Coleoptera, Dermestidae) fauny Palearktiki. Entomologischeskoe Obozrenie, 80: 611-620. 